\title{
Kalman Filtering for Discrete Time Uncertain Systems
}

\author{
Rodrigo Souto, João Ishihara and Geovany Borges \\ University of Brasília \\ Brazil
}

\section{Introduction}

State estimation plays an important role in any application dealing with modeling of dynamic systems. In fact, many fields of knowledge use a mathematical representation of a behavior of interest, such as, but not limited to, engineering (mechanical, electrical, aerospace, civil and chemical), physics, economics, mathematics and biology Simon (2006).

A typical system dynamics can be represented as a transfer function or using the space-state approach. The state-space approach is based on the time-evolution of the "states" of the system, which are considered all the necessary information to represent its dynamic at the desired point of operation. That is why the knowledge about the states of a model is so important. However, in real applications there can be two reasons where the states of a system can not be measured: a) measuring a state implies in the need of a sensor. In order to measure all the states of a system it will be required a large amount of sensors, making the project more expensive and sometimes unfeasible. Usually the whole cost includes not only the price of the sensors, but also modifies the project itself to fix all of them (engineering hours, more material to buy, a heavier product). b) Some states are impossible to be physically measured because they are a mathematically useful representation of the system, such as, the attitude parameterization of an aircraft altitude.

Suppose we have access to all the states of a system. What can we do with them? As the states contain all the information necessary about the system, one can use them to:

a) Implement a state-feedback controller Simon (2006). Almost in the same time the state estimation theory was being developed, the optimal control was growing in popularity mainly because its theory can guarantees closed loop stability margins. However, the Linear-Quadratic-Gaussian (LQG) control problem (the most fundamental optimal control problem) requires the knowledge of the states of the model, which motivated the development of the state estimation for those states that could not be measured in the plant to be controlled. b) Process monitoring. In this case, the knowledge of the state allows the monitoring of the system. This is very useful for navigation systems where it is necessary to know the position and the velocity of a vehicle, for instance, an aircraft or a submarine. In a radar system, this is its very purpose: keep tracking the position and velocity of all targets of interest in a given area. For an autonomous robot is very important to know its current position in relation to an inertial reference in order to keep it moving to its destiny. For a doctor is important to monitor the concentration of a given medicine in his patient. 
c) Process optimization. Once it is possible to monitor the system, the natural consequence is to make it work better. An actual application is the next generation of smart planes. Based on the current position and velocity of a set of aircraft, it is possible to a computer to better schedule arrivals, departures and routes in order to minimize the flight time, which also considers the waiting time for a slot in an airport to land the aircraft. Reducing the flight time means less fuel consumed, reducing the operation costs for the company and the environmental cost for the planet. Another application is based on the knowledge of the position and velocities of cell phones in a network, allowing an improved handover process (the process of transferring an ongoing call or data session from one channel connected to the core network to another), implying in a better connection for the user and smart network resource utilization.

d) Fault detection and prognostics. This is another immediate consequence of process monitoring. For example, suppose we are monitoring the current of an electrical actuator. In the case this current drops below a certain threshold we can conclude that the actuator is not working properly anymore. We have just detected a failure and a warning message can be sent automatically. In military application, this is essentially important when a system can be damaged by exterior reasons. Based on the knowledge of a failure occurrence, it is possible to switch the controller in order to try to overcome the failures. For instance, some aircraft prototypes were still able to fly and land after losing $60 \%$ of its wing. Thinking about the actuator system, but in a prognostics approach, we can monitor its current and note that it is dropping along the time. Usually, this is not an abrupt process: it takes so time to the current drop below its acceptable threshold. Based on the decreasing rate of the current, one is able to estimate when the actuator will stop working, and then replace it before it fails. This information is very important when we think about the safety of a system, preventing accidents in cars, aircrafts and other critical systems.

e) Reduce noise effect. Even in cases where the states are measured directly, state estimation schemes can be useful to reduce noise effect Anderson \& Moore (1979). For example, a telecommunication engineer wants to know the frequency and the amplitude of a sine wave received at his antenna. The environment and the hardware used may introduce some perturbations that disturb the sin wave, making the required measures imprecise. A state-state model of a sine wave and the estimation of its state can improve precision of the amplitude and frequency estimations.

When the states are not directly available, the above applications can still be performed by using estimates of the states. The most famous algorithm for state estimation is the Kalman filter Kalman (1960). It was initially developed in the 1960s and achieved a wide success to aerospace applications. Due its generic formulation, the same estimation theory could be applied to other practical fields, such as meteorology and economics, achieving the same success as in the aerospace industry. At our present time, the Kalman filter is the most popular algorithm to estimate the states of a system. Although its great success, there are some situations where the Kalman filter does not achieve good performance Ghaoui \& Clafiore (2001). The advances of technology lead to smaller and more sensible components. The degradation of these component became more often and remarkable. Also, the number and complexity of these components kept growing in the systems, making more and more difficult to model them all. Even if possible, it became unfeasible to simulate the system with these amounts of details. For these reasons (lack of dynamics modeling and more remarkable parameters changes), it became hard to provide the accurate models assumed by the Kalman. Also, in a lot of applications, it is not easy to obtain the required statistic information about 
noises and perturbations affecting the system. A new theory capable to deal with plant uncertainties was required, leading robust extensions of the Kalman filter. This new theory is referred as robust estimationGhaoui \& Clafiore (2001).

This chapter presents a robust prediction algorithm used to perform the state estimation of discrete time systems. The first part of the chapter describes how to model an uncertain system. In the following, the chapter presents the new robust technique used when dealing with linear inaccurate models. A numerical example is given to illustrate the advantages of using a robust estimator when dealing with an uncertain system.

\section{State Estimation}

The Estimation Theory was developed to solve the following problem: given the values of a observed signal though time, ${ }^{1}$ also known as measured signal, we require to estimate (smooth, correct or predict) the values of another signal that cannot be accessed directly or it is corrupted by noise or external perturbation.

The first step is to establish a relationship (or a model) between the measured and the estimated signal. Then we shall to define the criteria we will use to evaluate the model. In this sense, it is important to choose a criteria that is compatible with the model. The estimation is shown briefly at Figure 1.

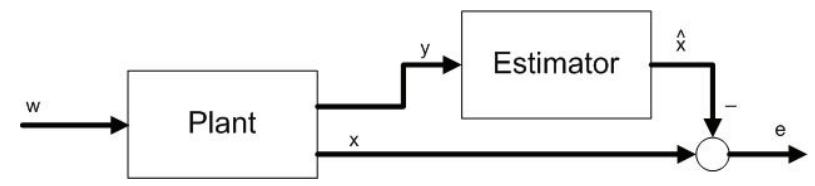

Fig. 1. Block diagram representing the estimation problem.

At Figure 1, we wish to estimate signal $x$. The signal $y$ are the measured values from the plant. The signal $w$ indicate an unknown input signal and it is usually represented by an stochastic behavior with known statistical properties. The estimation problem is about designing an algorithm that is able to provide $\hat{x}$, using the measures $y$, that are close of $x$ for several realizations of $y$. This same problem can also be classically formulated as a minimization of the estimation error variance. At the figure, the error is represented by $e$ and can be defined as $\hat{x}$ minus $x$. When we are dealing with a robust approach, our concern is to minimize an upper for the error variance as will be explained later on this chapter.

The following notation will be used along this chapter: $\mathbb{R}^{n}$ represents the $n$-dimensional Euclidean space, $\Re^{n \times m}$ is the set of real $n \times m$ matrices, $E\{\bullet\}$ denotes the expectation operator, $\operatorname{cov}\{\bullet\}$ stands for the covariance, $Z^{\dagger}$ represents the pseudo-inverse of the matrix $Z$, diag $\{\bullet\}$ stands for a block-diagonal matrix.

\section{Uncertain system modeling}

The following discrete-time model is a representation of a linear uncertain plant:

$$
\begin{aligned}
x_{k+1} & =A_{\Delta, k} x_{k}+\widetilde{w}_{k} \\
y_{k} & =C_{\Delta, k} x_{k}+\widetilde{v}_{k}
\end{aligned}
$$

\footnotetext{
${ }^{1}$ Signal here is used to define a data vector or a data set.
} 
where $x_{k} \in \mathbb{R}^{n_{x}}$ is the state vector, $y_{k} \in \mathbb{R}^{n_{y}}$ stands for the output vector and $\widetilde{w}_{k} \in \mathbb{R}^{n_{x}}$ and $\widetilde{v}_{k} \in \mathbb{R}^{n_{y}}$ are the output and measurement noises respectively. The uncertainties are characterized as:

1. Additive uncertainties at the dynamic represented as $A_{\Delta, k}=A_{k}+\Delta A_{k}$, where $A_{k}$ is the known, or expected, dynamic matrix and $\Delta A_{k}$ is the associated uncertainty.

2. Additive uncertainties at the output equation represented as $C_{\Delta, k}=C_{k}+\Delta C_{k}$, where $C_{k}$ is the known output matrix and $\Delta C_{k}$ characterizes its uncertainty.

3. Uncertainties at the mean, covariance and cross-covariance of the noises $\widetilde{w}_{k}$ and $\widetilde{v}_{k}$. We assume that the initial conditions $\left\{x_{0}\right\}$ and the noises $\left\{\widetilde{w}_{k}, \widetilde{v}_{k}\right\}$ are uncorrelated with the statistical properties

$$
\begin{aligned}
& E\left\{\left[\begin{array}{c}
\widetilde{w}_{k} \\
\widetilde{v}_{k} \\
x_{0}
\end{array}\right]\right\}=\left[\begin{array}{c}
E\left\{\widetilde{w}_{k}\right\} \\
E\left\{\widetilde{v}_{k}\right\} \\
\bar{x}_{0}
\end{array}\right], \\
& E\left\{\left[\begin{array}{c}
\widetilde{w}_{k}-E\left\{\widetilde{w}_{k}\right\} \\
\widetilde{v}_{k}-E\left\{\widetilde{v}_{k}\right\} \\
x_{0}-\bar{x}_{0}
\end{array}\right]\left[\begin{array}{c}
\widetilde{w}_{j}-E\left\{\widetilde{w}_{j}\right\} \\
\widetilde{v}_{j}-E\left\{\widetilde{v}_{j}\right\} \\
x_{0}-\bar{x}_{0}
\end{array}\right]\right\}=\left[\begin{array}{ccc}
\widetilde{W}_{k} \delta_{k j} & \widetilde{S}_{k} \delta_{k j} & 0 \\
\widetilde{S}_{k}^{T} \delta_{k j} & \widetilde{V}_{k} \delta_{k j} & 0 \\
0 & 0 & X_{0}
\end{array}\right],
\end{aligned}
$$

where $\widetilde{W}_{k}, \widetilde{V}_{k}$ and $X_{0}$ denotes the noises and initial state covariance matrices, $\widetilde{S}_{k}$ is the cross covariance and $\delta_{k j}$ is the Kronecker delta function.

Although the exact values of the means and of the covariances are unknown, it is assumed that they are within a known set. The notation at (5) will be used to represent the covariances sets.

$$
\widetilde{W}_{k} \in \mathcal{W}_{k}, \widetilde{V}_{k} \in \mathcal{V}_{k}, \widetilde{S}_{k} \in \mathcal{S}_{k} .
$$

In the next sub section, it will be presented how to characterize a system with uncertain covariance as a system with known covariance, but with uncertain parameters.

\subsection{The noises means and covariances spaces}

In this sub section, we will analyze some features of the noises uncertainties. The approach shown above considered correlated $\widetilde{w}_{k}$ and $\widetilde{v}_{k}$ with unknown mean, covariance and cross covariance, but within a known set. As will be shown later on, these properties can be achieved when we define the following noises structures:

$$
\begin{aligned}
\widetilde{w}_{k} & :=B_{\Delta w, k} w_{k}+B_{\Delta v, k} v_{k} \\
\widetilde{v}_{k} & :=D_{\Delta w, k} w_{k}+D_{\Delta v, k} v_{k} .
\end{aligned}
$$

Also here we assume that the initial conditions $\left\{x_{0}\right\}$ and the noises $\left\{w_{k}\right\},\left\{v_{k}\right\}$ are uncorrelated with the statistical properties

$$
\begin{aligned}
& E\left\{\left[\begin{array}{l}
w_{k} \\
v_{k} \\
x_{0}
\end{array}\right]\right\}=\left[\begin{array}{l}
\bar{w}_{k} \\
\bar{v}_{k} \\
\bar{x}_{0}
\end{array}\right], \\
& E\left\{\left[\begin{array}{c}
w_{k}-\bar{w}_{k} \\
v_{k}-\bar{v}_{k} \\
x_{0}-\bar{x}_{0}
\end{array}\right]\left[\begin{array}{c}
w_{j}-\bar{w}_{j} \\
v_{j}-\bar{v}_{j} \\
x_{0}-\bar{x}_{0}
\end{array}\right]\right\}=\left[\begin{array}{ccc}
W_{k} \delta_{k j} & S_{k} \delta_{k j} & 0 \\
S_{k}^{T} \delta_{k j} & V_{k} \delta_{k j} & 0 \\
0 & 0 & X_{0}
\end{array}\right],
\end{aligned}
$$


where $W_{k}, V_{k}$ and $X_{0}$ denotes the noises and initial state covariance matrices and $S_{k}$ stands for the cross covariance matrix of the noises.

Therefore using the properties (8) and (9) and the noises definitions (6) and (7), we can note that the noises $\widetilde{w}_{k}$ and $\widetilde{v}_{k}$ have uncertain mean given by

$$
\begin{aligned}
E\left\{\widetilde{w}_{k}\right\} & =B_{\Delta w, k} \bar{w}_{k}+B_{\Delta v, k} \bar{v}_{k \prime} \\
E\left\{\widetilde{v}_{k}\right\} & =D_{\Delta w, k} \bar{w}_{k}+D_{\Delta v, k} \bar{v}_{k} .
\end{aligned}
$$

Their covariances are also uncertain and given by

$$
E\left\{\left[\begin{array}{c}
\widetilde{w}_{k}-E\left\{\widetilde{w}_{k}\right\} \\
\widetilde{v}_{k}-E\left\{\widetilde{v}_{k}\right\}
\end{array}\right]\left[\begin{array}{c}
\widetilde{w}_{j}-E\left\{\widetilde{w}_{j}\right\} \\
\widetilde{v}_{j}-E\left\{\widetilde{v}_{j}\right\}
\end{array}\right]^{T}\right\}=\left[\begin{array}{c}
\widetilde{W}_{k} \delta_{k j} \widetilde{S}_{k} \delta_{k j} \\
\widetilde{S}_{k}^{T} \delta_{k j} \\
\widetilde{V}_{k} \delta_{k j}
\end{array}\right] .
$$

Using the descriptions (6) and (7) for the noises, we obtain

$$
\left[\begin{array}{cc}
\widetilde{W}_{k} \delta_{k j} & \widetilde{S}_{k} \delta_{k j} \\
\widetilde{S}_{k}^{T} \delta_{k j} & \widetilde{V}_{k} \delta_{k j}
\end{array}\right]=\left[\begin{array}{cc}
B_{\Delta w, k} & B_{\Delta v, k} \\
D_{\Delta w, k} & D_{\Delta v, k}
\end{array}\right]\left[\begin{array}{ll}
W_{k} \delta_{k j} & S_{k} \delta_{k j} \\
S_{k}^{T} \delta_{k j} & V_{k} \delta_{k j}
\end{array}\right]\left[\begin{array}{cc}
B_{\Delta w, k} & B_{\Delta v, k} \\
D_{\Delta w, k} & D_{\Delta v, k}
\end{array}\right]^{T} .
$$

The notation at (13) is able to represent noises with the desired properties of uncertain covariance and cross covariance. However we can consider some simplifications and achieve the same properties. There are two possible ways to simplify equation (13):

1. Set

$$
\left[\begin{array}{cc}
B_{\Delta w, k} & B_{\Delta v, k} \\
D_{\Delta w, k} & D_{\Delta v, k}
\end{array}\right]=\left[\begin{array}{cc}
B_{\Delta w, k} & 0 \\
0 & D_{\Delta v, k}
\end{array}\right]
$$

In this case, the covariance matrices can be represented as

$$
\left[\begin{array}{cc}
\widetilde{W}_{k} \delta_{k j} & \widetilde{S}_{k} \delta_{k j} \\
\widetilde{S}_{k}^{T} \delta_{k j} & \widetilde{V}_{k} \delta_{k j}
\end{array}\right]=\left[\begin{array}{cc}
B_{\Delta w, k} W_{k} B_{\Delta w, k}^{T} & B_{\Delta w, k} S_{k} D_{\Delta v, k}^{T} \\
D_{\Delta v, k} S_{k}^{T} B_{\Delta w, k}^{T} & D_{\Delta v, k} V_{k} D_{\Delta v, k}^{T}
\end{array}\right] \delta_{k j}
$$

2. The other approach is to consider

$$
\left[\begin{array}{ll}
W_{k} \delta_{k j} & S_{k} \delta_{k j} \\
S_{k}^{T} \delta_{k j} & V_{k} \delta_{k j}
\end{array}\right]=\left[\begin{array}{cc}
W_{k} \delta_{k j} & 0 \\
0 & V_{k} \delta_{k j}
\end{array}\right]
$$

In this case, the covariance matrices are given by

$$
\left[\begin{array}{cc}
\widetilde{W}_{k} \delta_{k j} & \widetilde{S}_{k} \delta_{k j} \\
\widetilde{S}_{k}^{T} \delta_{k j} & \widetilde{V}_{k} \delta_{k j}
\end{array}\right]=\left[\begin{array}{cc}
B_{\Delta w, k} W_{k} B_{\Delta w, k}^{T}+B_{\Delta v, k} V_{k} B_{\Delta v, k}^{T} & B_{\Delta w, k} W_{k} D_{\Delta w, k}^{T}+B_{\Delta v, k} V_{k} D_{\Delta v, k}^{T} \\
D_{\Delta w, k} W_{k} B_{\Delta w, k}^{T}+D_{\Delta v, k} V_{k} B_{\Delta v, k}^{T} & D_{\Delta w, k} W_{k} D_{\Delta w, k}^{T}+D_{\Delta v, k} V_{k} D_{\Delta v, k}^{T}
\end{array}\right] \delta_{k j} .
$$

So far we did not make any assumption about the structure of noises uncertainties at (6) and (7). As we did for the dynamic and the output matrices, it will be assumed additive uncertainties for the structure of the noises such as

$$
\begin{aligned}
& B_{\Delta w, k}:=B_{w, k}+\Delta B_{w, k}, \quad B_{\Delta v, k}:=B_{v, k}+\Delta B_{v, k} \\
& D_{\Delta w, k}:=D_{w, k}+\Delta D_{w, k}, \quad D_{\Delta v, k}:=D_{v, k}+\Delta D_{v, k}
\end{aligned}
$$


where $B_{w, k}, B_{v, k}, D_{w, k}$ and $D_{v, k}$ denote the nominal matrices. Their uncertainties are represented by $\Delta B_{w, k}, \Delta B_{v, k}, \Delta D_{w, k}$ and $\Delta D_{v, k}$ respectively. Using the structures (18)-(19) for the uncertainties, then we are able to obtain the following representation

$$
\begin{aligned}
\widetilde{w}_{k} & =\left(B_{w, k}+\Delta B_{w, k}\right) w_{k}+\left(B_{v, k}+\Delta B_{v, k}\right) v_{k} \\
\widetilde{v}_{k} & =\left(D_{w, k}+\Delta D_{w, k}\right) w_{k}+\left(D_{v, k}+\Delta D_{v, k}\right) v_{k} .
\end{aligned}
$$

In this case, we can note that the mean of the noises depend on the uncertain parameters of the model. The same applies to the covariance matrix.

\section{Linear robust estimation}

\subsection{Describing the model}

Consider the following class of uncertain systems presented at (1)-(2):

$$
\begin{aligned}
x_{k+1} & =\left(A_{k}+\Delta A_{k}\right) x_{k}+\widetilde{w}_{k} \\
y_{k} & =\left(C_{k}+\Delta C_{k}\right) x_{k}+\widetilde{v}_{k},
\end{aligned}
$$

where $x_{k} \in \mathbb{R}^{n_{x}}$ is the state vector, $y_{k} \in \mathbb{R}^{n_{y}}$ is the output vector and $\widetilde{w}_{k} \in \mathbb{R}^{n_{x}}$ and $\widetilde{v}_{k} \in$ $\mathbb{R}^{n_{y}}$ are noise signals. It is assumed that the noise signals $\widetilde{w}_{k}$ and $\widetilde{v}_{k}$ are correlated and their time-variant mean, covariance and cross-covariance are uncertain but within known bounded sets. We assume that these known sets are described as presented previously at (20)-(21) with the same statistical properties as (8)-(9).

Using the noise modeling (20) and (21), the system (22)-(23) can be written as

$$
\begin{aligned}
x_{k+1} & =\left(A_{k}+\Delta A_{k}\right) x_{k}+\left(B_{w, k}+\Delta B_{w, k}\right) w_{k}+\left(B_{v, k}+\Delta B_{v, k}\right) v_{k} \\
y_{k} & =\left(C_{k}+\Delta C_{k}\right) x_{k}+\left(D_{w, k}+\Delta D_{w, k}\right) w_{k}+\left(D_{v, k}+\Delta D_{v, k}\right) v_{k} .
\end{aligned}
$$

The dimensions are shown at Table (1).

\begin{tabular}{|c|l|}
\hline Matrix or vector & Set \\
\hline$x_{k}$ & $\mathbb{R}^{n_{x}}$ \\
$y_{k}$ & $\mathbb{R}^{n_{y}}$ \\
$w_{k}$ & $\mathbb{R}^{n_{w}}$ \\
$v_{k}$ & $\mathbb{R}^{n_{v}}$ \\
$A_{k}$ & $\mathbb{R}^{n_{x} \times n_{x}}$ \\
$B_{w, k}$ & $\mathbb{R}^{n_{x} \times n_{w}}$ \\
$B_{v, k}$ & $\mathbb{R}^{n_{x} \times n_{v}}$ \\
$C_{k}$ & $\mathbb{R}^{n_{y} \times n_{x}}$ \\
$D_{w, k}$ & $\mathbb{R}^{n_{y} \times n_{w}}$ \\
$D_{v, k}$ & $\mathbb{R}^{n_{y} \times n_{v}}$ \\
\hline
\end{tabular}

Table 1. Matrices and vectors dimensions.

The model (24)-(25) with direct feedthrough is equivalent to one with only one noise vector at the state and output equations and that $w_{k}$ and $v_{k}$ could have cross-covariance Anderson \& Moore (1979). However, we have preferred to use the redundant noise representation (20)-(21) with $w_{k}$ and $v_{k}$ uncorrelated in order to get a more accurate upper bound for the predictor covariance error. The nominal matrices $A_{k}, B_{w, k}, B_{v, k}, C_{k}, D_{w, k}$ and $D_{v, k}$ are known and the matrices $\Delta A_{k}, \Delta B_{w, k}, \Delta B_{v, k}, \Delta C_{k}, \Delta D_{w, k}$ and $\Delta D_{v, k}$ represent the associated uncertainties. 
The only assumptions we made on the uncertainties is that they are additive and are within a known set. In order to proceed the analysis it is necessary more information about the uncertainties. Usually the uncertainties are assumed norm bounded or within a polytope. The second approach requires more complex analysis, although the norm bounded set is within the set represented by a polytope.

In this chapter, it will be considered norm bounded uncertainties. For the general case, each uncertainty of the system can be represented as

$$
\begin{aligned}
\Delta A_{k} & :=H_{A, k} F_{A, k} G_{A, k} \\
\Delta B_{w, k} & :=H_{B w, k} F_{B w, k} G_{B w, k} \\
\Delta B_{v, k} & :=H_{B v, k} F_{B v, k} G_{B v, k} \\
\Delta C_{k} & :=H_{C, k} F_{C, k} G_{C, k} \\
\Delta D_{w, k} & :=H_{D w, k} F_{D w, k} G_{D w, k} \\
\Delta D_{v, k} & :=H_{D v, k} F_{D v, k} G_{D v, k} .
\end{aligned}
$$

where $H_{A, k}, H_{B w, k}, H_{B v, k}, H_{C, k}, H_{D w, k}, H_{D v, k}, G_{x, k}, G_{w, k}$ and $G_{v, k}$ are known. The matrices $F_{A, k}, F_{B w, k}, F_{B v, k}, F_{C, k}, F_{D w, k}$ and $F_{D v, k}$ are unknown, time varying and norm-bounded, i.e.,

$$
F_{A, k}^{T} F_{A, k} \leq I, F_{B w, k}^{T} F_{B w, k} \leq I, F_{B v, k}^{T} F_{B v, k} \leq I, F_{C, k}^{T} F_{C, k} \leq I, F_{D w, k}^{T} F_{D w, k} \leq I, F_{D v, k}^{T} F_{D v, k} \leq I .
$$

These uncertainties can also be represented at a matrix format as

$$
\begin{aligned}
& {\left[\begin{array}{ccc}
\Delta A_{k} & \Delta B_{w, k} & \Delta B_{v, k} \\
\Delta C_{k} & \Delta D_{w, k} & \Delta D_{v, k}
\end{array}\right]} \\
& =\left[\begin{array}{ccc}
H_{A, k} F_{A, k} G_{A, k} & H_{B w, k} F_{B w, k} G_{B w, k} & H_{B v, k} F_{B v, k} G_{B v, k} \\
H_{C, k} F_{C, k} G_{C, k} & H_{D w, k} F_{D w, k} G_{D w, k} & H_{D v, k} F_{D v, k} G_{D v, k}
\end{array}\right] \\
& =\left[\begin{array}{cccccc}
H_{A, k} & H_{B w, k} & H_{B v, k} & 0 & 0 & 0 \\
0 & 0 & 0 & H_{C, k} & H_{D w, k} & H_{D v, k}
\end{array}\right] \\
& \times \operatorname{diag}\left\{F_{A, k}, F_{B w, k}, F_{B v, k}, F_{C, k}, F_{D w, k}, F_{D v, k}\right\}\left[\begin{array}{ccc}
G_{A, k} & 0 & 0 \\
0 & G_{B w, k} & 0 \\
0 & 0 & G_{B v, k} \\
G_{C, k} & 0 & 0 \\
0 & G_{D w, k} & 0 \\
0 & 0 & G_{D v, k}
\end{array}\right] .
\end{aligned}
$$

However, there is another way to represent distinct uncertainties for each matrix by the appropriate choice of the matrices $H$ as follows

$$
\begin{aligned}
{\left[\begin{array}{l}
\Delta A_{k} \\
\Delta C_{k}
\end{array}\right] } & :=\left[\begin{array}{l}
H_{A, k} \\
H_{C, k}
\end{array}\right] F_{x, k} G_{x, k} \\
{\left[\begin{array}{c}
\Delta B_{w, k} \\
\Delta D_{w, k}
\end{array}\right] } & :=\left[\begin{array}{l}
H_{B w, k} \\
H_{D w, k}
\end{array}\right] F_{w, k} G_{w, k} \\
{\left[\begin{array}{c}
\Delta B_{v, k} \\
\Delta D_{v, k}
\end{array}\right] } & :=\left[\begin{array}{l}
H_{B v, k} \\
H_{D v, k}
\end{array}\right] F_{v, k} G_{v, k}
\end{aligned}
$$


where the matrices $F_{x, k}, F_{w, k}$ and $F_{v, k}$ of dimensions $r_{x, k} \times s_{x, k}, r_{w, k} \times s_{w, k}, r_{v, k} \times s_{v, k}$ are unknown and norm-bounded, $\forall k \in[0, N]$, i.e.,

$$
F_{x, k}^{T} F_{x, k} \leq I, F_{w, k}^{T} F_{w, k} \leq I, F_{v, k}^{T} F_{v, k} \leq I .
$$

Rewriting the uncertainties into a matrix structure, we obtain

$$
\begin{aligned}
& {\left[\begin{array}{ccc}
\Delta A_{k} & \Delta B_{w, k} & \Delta B_{v, k} \\
\Delta C_{k} & \Delta D_{w, k} & \Delta D_{v, k}
\end{array}\right]=\left[\begin{array}{ccc}
H_{A, k} F_{x, k} G_{x, k} & H_{B w, k} F_{w, k} G_{w, k} & H_{B v, k} F_{v, k} G_{v, k} \\
H_{C, k} F_{x, k} G_{x, k} & H_{D w, k} F_{w, k} G_{w, k} & H_{D v, k} F_{v, k} G_{v, k}
\end{array}\right]} \\
& =\left[\begin{array}{lll}
H_{A, k} & H_{B w, k} & H_{B v, k} \\
H_{C, k} & H_{D w, k} & H_{D v, k}
\end{array}\right]\left[\begin{array}{ccc}
F_{x, k} & 0 & 0 \\
0 & F_{w, k} & 0 \\
0 & 0 & F_{v, k}
\end{array}\right]\left[\begin{array}{ccc}
G_{x, k} & 0 & 0 \\
0 & G_{w, k} & 0 \\
0 & 0 & G_{v, k}
\end{array}\right] \text {. }
\end{aligned}
$$

Our goal is to design a finite horizon robust predictor for state estimation of the uncertain system described by (24)-(37). We consider predictors with the following structure

$$
\begin{aligned}
\widehat{x}_{0 \mid-1} & =\bar{x}_{0}, \\
\widehat{x}_{k+1 \mid k} & =\Phi_{k} \widehat{x}_{k \mid k-1}+B_{w, k} \bar{w}_{k}+B_{v, k} \bar{v}_{k}+K_{k}\left(y_{k}-C_{k} \widehat{x}_{k \mid k-1}-D_{w, k} \bar{w}_{k}-D_{v, k} \bar{v}_{k}\right) .
\end{aligned}
$$

The predictor is intended to ensure an upper limit in the error estimation variance. In other words, we seek a sequence of non-negative definite matrices $\left\{\bar{P}_{k+1 \mid k}\right\}$ that, for all allowed uncertainties, satisfy for each $k$

$$
\operatorname{cov}\left\{e_{k+1 \mid k}\right\} \leq \bar{P}_{k+1 \mid k}
$$

where $e_{k+1 \mid k}=x_{k+1}-\widehat{x}_{k+1 \mid k}$. The matrices $\Phi_{k}$ and $K_{k}$ are time-varying and shall be determined in such way that the upper bound $\bar{P}_{k+1 \mid k}$ is minimized.

\subsection{A robust estimation solution}

At this part, we shall choose an augmented state vector. There are normally found two options are found in the literature:

$$
\tilde{x}_{k}:=\left[\begin{array}{c}
x_{k} \\
\widehat{x}_{k \mid k-1}
\end{array}\right], \tilde{x}_{k}:=\left[\begin{array}{c}
x_{k}-\widehat{x}_{k \mid k-1} \\
\widehat{x}_{k \mid k-1}
\end{array}\right] .
$$

One can note that there is a similarity transformation between both vectors. This transformation matrix and its inverse are given by

$$
T=\left[\begin{array}{ll}
I & I \\
0 & I
\end{array}\right], T^{-1}=\left[\begin{array}{cc}
I & -I \\
0 & I
\end{array}\right] .
$$

Using the system definition (24)-(25) and the structure of the estimator in (40) then we define an augmented system as

$$
\begin{aligned}
\widetilde{x}_{k+1} & =\left(\widetilde{A}_{k}+\widetilde{H}_{x, k} F_{x, k} \widetilde{G}_{x, k}\right) \widetilde{x}_{k}+\left(\widetilde{B}_{k}+\widetilde{H}_{w, k} F_{w, k} G_{w, k}\right) w_{k}+\widetilde{\bar{B}}_{k} \bar{w}_{k} \\
& +\left(\widetilde{D}_{k}+\widetilde{H}_{v, k} F_{v, k} G_{v, k}\right) v_{k}+\widetilde{\bar{D}}_{k} \bar{v}_{k}
\end{aligned}
$$


where

$$
\begin{aligned}
& \widetilde{D}_{k}=\left[\begin{array}{c}
B_{v, k} \\
K_{k} D_{v, k}
\end{array}\right], \widetilde{H}_{v, k}=\left[\begin{array}{c}
H_{B v, k} \\
K_{k} H_{D v, k}
\end{array}\right], \widetilde{G}_{x, k}=\left[\begin{array}{l}
G_{x, k} 0
\end{array}\right], \\
& \widetilde{B}_{k}=\left[\begin{array}{c}
B_{w, k} \\
K_{k} D_{w, k}
\end{array}\right], \widetilde{H}_{w, k}=\left[\begin{array}{c}
H_{B w, k} \\
K_{k} H_{D w, k}
\end{array}\right], \widetilde{x}_{k}=\left[\begin{array}{c}
x_{k} \\
\widehat{x}_{k \mid k-1}
\end{array}\right], \\
& \widetilde{A}_{k}=\left[\begin{array}{cc}
A_{k} & 0 \\
K_{k} C_{k} & \Phi_{k}-K_{k} C_{k}
\end{array}\right], \widetilde{H}_{x, k}=\left[\begin{array}{c}
H_{A, k} \\
K_{k} H_{C, k}
\end{array}\right], \\
& \widetilde{\bar{B}}_{k}=\left[\begin{array}{c}
0 \\
B_{w, k}-K_{k} D_{w, k}
\end{array}\right], \widetilde{\bar{D}}_{k}=\left[\begin{array}{c}
0 \\
B_{v, k}-K_{k} D_{v, k}
\end{array}\right] \text {. }
\end{aligned}
$$

Consider $\widetilde{P}_{k+1 \mid k}=\operatorname{cov}\left\{\widetilde{x}_{k+1}\right\}$. The next lemma give us an upper bound for the covariance matrix of the augmented system (44).

Lemma 1. An upper limit for the covariance matrix of the augmented system (44) is given by $P_{0 \mid-1}=\operatorname{diag}\left\{X_{0}, 0\right\}$ and

$$
\begin{aligned}
P_{k+1 \mid k} & =\widetilde{A}_{k} P_{k \mid k-1} \widetilde{A}_{k}^{T}+\widetilde{B}_{k} W_{c, k} \widetilde{B}_{k}^{T}+\widetilde{D}_{k} V_{c, k} \widetilde{D}_{k}^{T} \\
& +\widetilde{A}_{k} P_{k \mid k-1} \widetilde{G}_{x, k}^{T}\left(\alpha_{x, k}^{-1} I-\widetilde{G}_{x, k} P_{k \mid k-1} \widetilde{G}_{x, k}^{T}\right)^{-1} \widetilde{G}_{x, k} P_{k \mid k-1} \widetilde{A}_{k}^{T} \\
& +\alpha_{x, k}^{-1} \widetilde{H}_{x, k} \widetilde{H}_{x, k}^{T}+\alpha_{w, k}^{-1} \widetilde{H}_{w, k} \widetilde{H}_{w, k}^{T}+\alpha_{v, k}^{-1} \widetilde{H}_{v, k} \widetilde{H}_{v, k}^{T}
\end{aligned}
$$

where $\alpha_{x, k}^{-1}, \alpha_{w, k}^{-1}$ and $\alpha_{v, k}^{-1}$ satisfy

$$
\begin{aligned}
& \alpha_{x, k}^{-1} I-\widetilde{G}_{x, k} P_{k \mid k-1} \widetilde{G}_{x, k}^{T}>0, \\
& \alpha_{w, k}^{-1} I-G_{w, k} W_{k} G_{w, k}^{T}>0, \\
& \alpha_{v, k}^{-1} I-G_{v, k} V_{k} G_{v, k}^{T}>0 .
\end{aligned}
$$

Proof: Since $\widetilde{x}_{k}, w_{k}$ and $v_{k}$ are uncorrelated signals, and using (8), (9), (39) and (44), it is straightforward that $\widetilde{P}_{0 \mid-1}=\operatorname{diag}\left\{X_{0}, 0\right\}$ and

$$
\begin{aligned}
\widetilde{P}_{k+1 \mid k} & =\left(\widetilde{A}_{k}+\widetilde{H}_{x, k} F_{x, k} \widetilde{G}_{x, k}\right) \widetilde{P}_{k \mid k-1}\left(\widetilde{A}_{k}+\widetilde{H}_{x, k} F_{x, k} \widetilde{G}_{x, k}\right)^{T} \\
& +\left(\widetilde{B}_{k}+\widetilde{H}_{w, k} F_{w, k} G_{w, k}\right) W_{k}\left(\widetilde{B}_{k}+\widetilde{H}_{w, k} F_{w, k} G_{w, k}\right)^{T} \\
& +\left(\widetilde{D}_{k}+\widetilde{H}_{v, k} F_{v, k} G_{v, k}\right) V_{k}\left(\widetilde{D}_{k}+\widetilde{H}_{v, k} F_{v, k} G_{v, k}\right)^{T} .
\end{aligned}
$$

Choose scaling parameters $\alpha_{x, k^{\prime}}^{-1}, \alpha_{w, k}^{-1}$ and $\alpha_{v, k}^{-1}$ satisfying (47)-(49). Using Lemma 2 of Wang et al. (1999) and Lemma 3.2 of Theodor \& Shaked (1996), we have that the sequence $\left\{P_{k+1 \mid k}\right\}$ given by (46) is such that $\widetilde{P}_{k+1 \mid k} \leq P_{k+1 \mid k}$ for all instants $k$. QED.

Replacing the augmented matrices (45) into (46), the upper bound $P_{k+1 \mid k}$ can be partitioned as

$$
P_{k+1 \mid k}=\left[\begin{array}{ll}
P_{11, k+1 \mid k} & P_{12, k+1 \mid k} \\
P_{12, k+1 \mid k}^{T} & P_{22, k+1 \mid k}
\end{array}\right],
$$


where, using the definitions presented in Step 1 of Table 2, we obtain

$$
\begin{aligned}
P_{11, k+1 \mid k} & =A_{k} P_{11 c, k} A_{k}^{T}+B_{k} U_{c, k} B_{k}^{T}+\Delta_{3, k} \\
P_{12, k+1 \mid k} & =A_{k} P_{12 c, k} \Phi_{k}^{T}+A_{k} S_{1, k} C_{k}^{T} K_{k}^{T}+\left(B_{k} U_{c, k} D_{k}^{T}+\Delta_{1, k}\right) K_{k}^{T}, \\
P_{22, k+1 \mid k} & =\Phi_{k} P_{22 c, k} \Phi_{k}^{T}+K_{k} C_{k} S_{2, k} \Phi_{k}^{T}+\Phi_{k} S_{2, k}^{T} C_{k}^{T} K_{k}^{T} \\
& +K_{k}\left(C_{k} S_{3, k} C_{k}^{T}+D_{k} U_{c, k} D_{k}^{T}+\Delta_{2, k}\right) K_{k}^{T}
\end{aligned}
$$

with

$$
\begin{aligned}
U_{c, k} & :=\left[\begin{array}{cc}
W_{c, k} & 0 \\
0 & V_{c, k}
\end{array}\right], \\
\Delta_{1, k} & :=\alpha_{x, k}^{-1} H_{A, k} H_{C, k}^{T}+\alpha_{w, k}^{-1} H_{B w, k} H_{D w, k}^{T}+\alpha_{v, k}^{-1} H_{B v, k} H_{D v, k^{\prime}}^{T} \\
\Delta_{2, k} & :=\alpha_{x, k}^{-1} H_{C, k} H_{C, k}^{T}+\alpha_{w, k}^{-1} H_{D w, k} H_{D w, k}^{T}+\alpha_{v, k}^{-1} H_{D v, k} H_{D v, k}^{T}, \\
\Delta_{3, k} & :=\alpha_{x, k}^{-1} H_{A, k} H_{A, k}^{T}+\alpha_{w, k}^{-1} H_{B w, k} H_{B w, k}^{T}+\alpha_{v, k}^{-1} H_{B v, k} H_{B v, k \prime}^{T} \\
M_{k} & :=G_{x, k}^{T}\left(\alpha_{x, k}^{-1} I-G_{x, k} P_{11, k \mid k-1} G_{x, k}^{T}\right)^{-1} G_{x, k}, \\
P_{11 c, k} & :=P_{11, k \mid k-1}+P_{11, k \mid k-1} M_{k} P_{11, k \mid k-1}, \\
P_{12 c, k} & :=P_{12, k \mid k-1}+P_{11, k \mid k-1} M_{k} P_{12, k \mid k-1}, \\
P_{22 c, k} & :=P_{22, k \mid k-1}+P_{12, k \mid k-1}^{T} M_{k} P_{12, k \mid k-1}, \\
S_{1, k} & :=P_{11 c, k}-P_{12 c, k} \\
S_{2, k} & :=P_{12 c, k}-P_{22 c, k} \\
S_{3, k} & :=S_{1, k}-S_{2, k}^{T} .
\end{aligned}
$$

Since $P_{k+1 \mid k} \geq \widetilde{P}_{k+1 \mid k} \geq 0, \forall k$, it is clear that if we define

$$
\bar{P}_{k+1 \mid k}=\left[\begin{array}{ll}
I & -I
\end{array}\right] P_{k+1 \mid k}\left[\begin{array}{ll}
I & -I
\end{array}\right]^{T},
$$

then we have that $\bar{P}_{k+1 \mid k}$ is an upper bound of the error variance on the state estimation. Using the definitions (50) and (65), the initial condition for $\bar{P}_{k+1 \mid k}$ is $\bar{P}_{0 \mid-1}=X_{0}$ and $\bar{P}_{k+1 \mid k}$ can be written as

$$
\begin{aligned}
\bar{P}_{k+1 \mid k} & =\left(A_{k}-K_{k} C_{k}\right) P_{11, c}\left(A_{k}-K_{k} C_{k}\right)^{T}-\left(A_{k}-K_{k} C_{k}\right) P_{12, c}\left(\Phi_{k}-K_{k} C_{k}\right)^{T} \\
& -\left(\Phi_{k}-K_{k} C_{k}\right) P_{12, c}^{T}\left(A_{k}-K_{k} C_{k}\right)^{T}+\left(\Phi_{k}-K_{k} C_{k}\right) P_{22, c 1}\left(\Phi_{k}-K_{k} C_{k}\right)^{T} \\
& +\left(B_{w, k}-K_{k} D_{w, k}\right) W_{c, k}\left(B_{w, k}-K_{k} D_{w, k}\right)^{T} \\
& +\left(B_{v, k}-K_{k} D_{v, k}\right) V_{c, k}\left(B_{v, k}-K_{k} D_{v, k}\right)^{T} \\
& +\alpha_{x, k}^{-1}\left(H_{A, k}-K_{k} H_{C, k}\right)\left(H_{A, k}-{ }_{k} H_{C, k}\right)^{T} \\
& +\alpha_{w, k}^{-1}\left(H_{B w, k}-K_{k} H_{D w, k}\right)\left(H_{B w, k}-K_{k} H_{D w, k}\right)^{T} \\
& +\alpha_{v, k}^{-1}\left(H_{B v, k}-K_{k} H_{D v, k}\right)\left(H_{B v, k}-K_{k} H_{D v, k}\right)^{T} .
\end{aligned}
$$


Note that $\bar{P}_{k+1 \mid k}$ given by (66) satisfies (41) for any $\Phi_{k}$ and $K_{k}$. In this sense, we can choose them to minimize the covariance of the estimation error given by $\bar{P}_{k+1 \mid k}$. We calculate the first order partial derivatives of (66) with respect to $\Phi_{k}$ and $K_{k}$ and making them equal to zero, i.e.,

$$
\begin{gathered}
\frac{\partial}{\partial \Phi_{k}} \bar{P}_{k+1 \mid k}=0 \\
\frac{\partial}{\partial K_{k}} \bar{P}_{k+1 \mid k}=0 .
\end{gathered}
$$

Then the optimal values $\Phi_{k}=\Phi_{k}^{*}$ and $K_{k}=K_{k}^{*}$ are given by

$$
\begin{aligned}
K_{k}^{*} & =\left(A_{k} S_{k} C_{k}^{T}+\Psi_{1, k}\right)\left(C_{k} S_{k} C_{k}^{T}+\Psi_{2, k}\right)^{\dagger}, \\
\Phi_{k}^{*} & =A_{k}+\left(A_{k}-K_{k}^{*} C_{k}\right)\left(P_{12 c, k} P_{22 c, k}^{+}-I\right),
\end{aligned}
$$

where

$$
\begin{aligned}
S_{k} & :=P_{11 c, k}-P_{12 c, k} P_{22 c, k}^{\dagger} P_{12 c, k \prime}^{T} \\
\Psi_{1, k} & :=B_{w, k} W_{c, k} D_{w, k}^{T}+B_{v, k} V_{c, k} D_{v, k}^{T}+\Delta_{1, k} \\
\Psi_{2, k} & :=D_{w, k} W_{c, k} D_{w, k}^{T}+D_{v, k} V_{c, k} D_{v, k}^{T}+\Delta_{2, k} .
\end{aligned}
$$

Actually $\Phi_{k}^{*}$ and $K_{k}^{*}$ provide the global minimum of $\bar{P}_{k+1 \mid k}$. This can be proved though the convexity of $\bar{P}_{k+1 \mid k}$ at (66). We first have that $\widetilde{P}_{k+1 \mid k}>0, W_{k}>0$ and $V_{k}>0, \forall k$. Then we calculate the Hessian matrix to conclude that we have the global minimum:

$$
H e\left(\bar{P}_{k+1 \mid k}\right):=\left[\begin{array}{cc}
\frac{\partial^{2}}{\partial^{2} \Phi_{k}} \bar{P}_{k+1 \mid k} & \frac{\partial^{2}}{\partial^{2}\left[\Phi_{k}, K_{k}\right]} \bar{P}_{k+1 \mid k} \\
\frac{\partial^{2}}{\partial^{2}\left[K_{k}, \Phi_{k}\right]} \bar{P}_{k+1 \mid k} & \frac{\partial^{2}}{\partial^{2} K_{k}} \bar{P}_{k+1 \mid k}
\end{array}\right]=\left[\begin{array}{cc}
2 P_{22, k \mid k-1} & 2 C_{k} S_{2, k} \\
2 S_{2, k}^{T} C_{k}^{T} & C_{k} S_{k} C_{k}^{T}+\Psi_{3, k}
\end{array}\right]>0 .
$$

At the previous equations we used the pseudo-inverse instead of the simple matrix inverse. Taking a look at the initial conditions $P_{12,0 \mid-1}=P_{12,0 \mid-1}^{T}=P_{22,0 \mid-1}=0$, one can note that $P_{22,0}=0$ and, as consequence, the inverse does not exist for all instant $k$. However, it can be proved that the pseudo-inverse does exist.

Replacing (70) and (69) in (52) and (53), we obtain

$$
\begin{aligned}
& P_{12, k+1 \mid k}=P_{12, k+1 \mid k}^{T}=P_{22, k+1 \mid k}= \\
= & A_{k} P_{12 c, k} P_{22 c, k}^{-1} P_{12 c, k}^{T} A_{k}^{T}+\left(A_{k} S_{k} C_{k}^{T}+\Psi_{1, k}\right)\left(C_{k} S_{k} C_{k}^{T}+\Psi_{2, k}\right)^{\dagger}\left(A_{k} S_{k} C_{k}^{T}+\Psi_{1, k}\right)^{T} .
\end{aligned}
$$

Since (74) holds for any symmetric $P_{k+1 \mid k}$, if we start with a matrix $P_{n+1 \mid n}$ satisfying $P_{12, n+1 \mid n}=$ $P_{12, n+1 \mid n}^{T}=P_{22, n+1 \mid n}$ for some $n \geq 0$, then we can conclude that (74) is valid for any $k \geq n$. The equality allows us some simplifications. The first one is

$$
S_{k}=\bar{P}_{c, k \mid k-1}:=\bar{P}_{k \mid k-1}+\bar{P}_{k \mid k-1} G_{x, k}^{T}\left(\alpha_{x, k}^{-1} I-G_{x, k} \bar{P}_{k \mid k-1} G_{x, k}^{T}\right)^{-1} G_{x, k} \bar{P}_{k \mid k-1}
$$

In fact, the covariance matrix of the estimation error presents a modified notation to deal with the uncertain system. At this point, we can conclude that $\alpha_{x, k}$ shall now satisfy

$$
\alpha_{x, k}^{-1} I-G_{x, k} \bar{P}_{k \mid k-1} G_{x, k}^{T}>0 .
$$


Using (74), we can simplify the expressions for $\Phi_{k}^{*}, K_{k}^{*}$ and $\bar{P}_{k+1 \mid k}$. We can define $\Phi_{k}$ given in Step 4 of Table 2 as $\Phi_{k}=\Phi_{k}^{*}$. The simplified expression for the predictor gain is given by

$$
K_{k}^{*}=\left(A_{k} \bar{P}_{c, k \mid k-1} C_{k}^{T}+\Psi_{1, k}\right)\left(C_{k} \bar{P}_{c, k \mid k-1} C_{k}^{T}+\Psi_{2, k}\right)^{\dagger},
$$

which can be rewritten as presented in Step 4 of Table 2. The expression for the Riccati equation can be written as

$$
\begin{aligned}
\bar{P}_{k+1 \mid k} & =\left(A_{k}-K_{k}^{*} C_{k}\right) \bar{P}_{c, k \mid k-1}\left(A_{k}-K_{k}^{*} C_{k}\right)^{T} \\
& +\left(B_{w, k}-K_{k}^{*} D_{w, k}\right) W_{c, k}\left(B_{w, k}-K_{k}^{*} D_{w, k}\right)^{T} \\
& +\left(B_{v, k}-K_{k}^{*} D_{v, k}\right) V_{c, k}\left(B_{v, k}-K_{k}^{*} D_{v, k}\right)^{T} \\
& +\alpha_{x, k}^{-1}\left(H_{A, k}-K_{k}^{*} H_{C, k}\right)\left(H_{A, k}-K_{k}^{*} H_{C, k}\right)^{T} \\
& +\alpha_{w, k}^{-1}\left(H_{B w, k}-K_{k}^{*} H_{D w, k}\right)\left(H_{B w, k}-K_{k}^{*} H_{D w, k}\right)^{T} \\
& +\alpha_{v, k}^{-1}\left(H_{B v, k}-K_{k}^{*} H_{D v, k}\right)\left(H_{B v, k}-K_{k}^{*} H_{D v, k}\right)^{T} .
\end{aligned}
$$

Replacing the expression for $K_{k}^{*}$ in $\bar{P}_{k+1 \mid k}$, we obtain the Riccati equation given in Step 5 of Table 2.

Using an alternative representation, remember the predictor structure:

$$
\widehat{x}_{k+1 \mid k}=\Phi_{k} \widehat{x}_{k \mid k-1}+B_{w, k} \bar{w}_{k}+B_{v, k} \bar{v}_{k}+K_{k}\left(y_{k}-C_{k} \widehat{x}_{k \mid k-1}-D_{w, k} \bar{w}_{k}-D_{v, k} \bar{v}_{k}\right) .
$$

Replace $\Phi_{k}^{*}$ into (77) to obtain

$$
\widehat{x}_{k+1 \mid k}=A_{c, k} \widehat{x}_{k \mid k-1}+B_{w, k} \bar{w}_{k}+B_{v, k} \bar{v}_{k}+K_{k}\left(y_{k}-C_{c, k} \widehat{x}_{k \mid k-1}-D_{w, k} \bar{w}_{k}-D_{v, k} \bar{v}_{k}\right),
$$

where

$$
\begin{aligned}
& A_{c, k}:=A_{k}+A_{k} \bar{P}_{k \mid k-1} G_{x, k}^{T}\left(\alpha_{x, k}^{-1} I-G_{x, k} \bar{P}_{k \mid k-1} G_{x, k}^{T}\right)^{-1} G_{x, k} \\
& C_{c, k}:=C_{k}+C_{k} \bar{P}_{k \mid k-1} G_{x, k}^{T}\left(\alpha_{x, k}^{-1} I-G_{x, k} \bar{P}_{k \mid k-1} G_{x, k}^{T}\right)^{-1} G_{x, k} .
\end{aligned}
$$

Once again, it is possible to obtain the classic estimator from the structure (79)-(80) for a system without uncertainties.

\section{Numerical example}

At this section we perform a simulation to illustrate to importance to consider the uncertainties at your predictor design.

One good way to quantify the performance of the estimator would be using its real variance to the error estimation. However, this is difficult to obtain from the response of the model. For this reason, we approximate the real variance of the estimation error using the ensemble-average (see Ishihara et al. (2006) and Sayed (2001)) given by:

$$
\begin{aligned}
\operatorname{var}\left\{e_{i, k}\right\} & \approx \frac{1}{N} \sum_{j=1}^{N}\left(e_{i, k}^{(j)}-E\left\{e_{i, k}^{(j)}\right\}\right)^{2}, \\
E\left\{e_{i, k}^{(j)}\right\} & \approx \frac{1}{N} \sum_{j=1}^{N} e_{i, k}^{(j)}
\end{aligned}
$$


Step 0 (Initial conditions): $\widehat{x}_{0 \mid-1}=\bar{x}_{0}$ and $\bar{P}_{0 \mid-1}=X_{0}$.

Step 1: Obtain scalar parameters $\alpha_{x, k}, \alpha_{w, k}$ and $\alpha_{v, k}$ that satisfy (76),

(48) and (49), respectively. Then define

$\Delta_{1, k}:=\alpha_{x, k}^{-1} H_{A, k} H_{C, k}^{T}+\alpha_{w, k}^{-1} H_{B w, k} H_{D w, k}^{T}+\alpha_{v, k}^{-1} H_{B v, k} H_{D v, k^{\prime}}^{T}$

$\Delta_{2, k}:=\alpha_{x, k}^{-1} H_{C, k} H_{C, k}^{T}+\alpha_{w, k}^{-1} H_{D w, k} H_{D w, k}^{T}+\alpha_{v, k}^{-1} H_{D v, k} H_{D v, k}^{T}$

$\Delta_{3, k}:=\alpha_{x, k}^{-1} H_{A, k} H_{A, k}^{T}+\alpha_{w, k}^{-1} H_{B w, k} H_{B w, k}^{T}+\alpha_{v, k}^{-1} H_{B v, k} H_{B v, k}^{T}$.

Step 2: Calculate the corrections due to the presence of uncertainties

$$
\begin{aligned}
& \bar{P}_{c, k \mid k-1}:=\bar{P}_{k \mid k-1}+\bar{P}_{k \mid k-1} G_{x, k}^{T}\left(\alpha_{x, k}^{-1} I-G_{x, k} \bar{P}_{k \mid k-1} G_{x, k}^{T}\right)^{-1} G_{x, k} \bar{P}_{k \mid k-1}, \\
& W_{c, k}:=W_{k}+W_{k} G_{w, k}^{T}\left(\alpha_{w, k}^{-1} I-G_{w, k} W_{k} G_{w, k}^{T}\right)^{-1} G_{w, k} W_{k} . \\
& V_{c, k}:=V_{k}+V_{k} G_{v, k}^{T}\left(\alpha_{v, k}^{-1} I-G_{v, k} V_{k} G_{v, k}^{T}\right)^{-1} G_{v, k} V_{k},
\end{aligned}
$$

Step 3: Define the augmented matrices

$$
B_{k}:=\left[\begin{array}{ll}
B_{w, k} & B_{v, k}
\end{array}\right], D_{k}:=\left[\begin{array}{ll}
D_{w, k} & D_{v, k}
\end{array}\right], U_{c, k}:=\operatorname{diag}\left\{W_{c, k}, V_{c, k}\right\} .
$$

Step 4: Calculate the parameters of the predictor as

$$
\begin{aligned}
& K_{k}=\left(A_{k} \bar{P}_{c, k \mid k-1} C_{k}^{T}+B_{k} U_{c, k} D_{k}^{T}+\Delta_{1, k}\right)\left(C_{k} \bar{P}_{c, k \mid k-1} C_{k}^{T}+D_{k} U_{c, k} D_{k}^{T}+\Delta_{2, k}\right)^{\dagger}, \\
& \Phi_{k}=A_{k}+\left(A_{k}-K_{k} C_{k}\right) \bar{P}_{k \mid k-1} G_{x, k}^{T}\left(\alpha_{x, k}^{-1} I-G_{x, k} \bar{P}_{k \mid k-1} G_{x, k}^{T}\right)^{-1} G_{x, k} .
\end{aligned}
$$

Step 5: Update $\left\{\widehat{x}_{k+1 \mid k}\right\}$ and $\left\{\bar{P}_{k+1 \mid k}\right\}$ as

$$
\begin{aligned}
\widehat{x}_{k+1 \mid k} & =\Phi_{k} \widehat{x}_{k \mid k-1}+B_{w, k} \bar{w}_{k}+B_{v, k} \bar{v}_{k}+K_{k}\left(y_{k}-C_{k} \widehat{x}_{k \mid k-1}-D_{w, k} \bar{w}_{k}-D_{v, k} \bar{v}_{k}\right), \\
\bar{P}_{k+1 \mid k} & =A_{k} \bar{P}_{c, k \mid k-1} A_{k}^{T}+B_{k} U_{c, k} B_{k}^{T}+\Delta_{3, k} \\
& -\left(A_{k} \bar{P}_{c, k \mid k-1} C_{k}^{T}+\Delta_{1, k}\right)\left(C_{k} \bar{P}_{c, k \mid k-1} C_{k}^{T}+D_{k} U_{c, k} D_{k}^{T}+\Delta_{2, k}\right)^{\dagger}\left(A_{k} \bar{P}_{c, k \mid k-1} C_{k}^{T}+\Delta_{1, k}\right)^{T}
\end{aligned}
$$

Table 2. The Enhanced Robust Predictor.

where $e_{i, k}^{(j)}$ is the $i$-th component of the estimation error vector $e_{k}^{(j)}$ of the realization $j$ defined as

$$
e_{k}^{(j)}:=x_{k}^{(j)}-\widehat{x}_{k \mid k-1}^{(j)}
$$

Another way to quantify the performance of the estimation is though covariance ellipses. The use of covariance ellipses allows us to visualize the variance and the cross covariance of a system with two states. 
Consider the benchmark model, used for instance in Fu et al. (2001) and Theodor \& Shaked (1996), where we added uncertainties in order to affect every matrix of the system,

$$
\begin{aligned}
x_{k+1} & =\left[\begin{array}{cc}
0 & -0.5 \\
1+\delta_{x, k} & 1+0.3 \delta_{x, k}
\end{array}\right] x_{k}+\left[\begin{array}{c}
-6 \\
1+0.1 \delta_{w, k}
\end{array}\right] w_{k}, \\
y_{k} & =\left[\begin{array}{ll}
-100+5 \delta_{x, k} & 10+1.5 \delta_{x, k}
\end{array}\right] x_{k}+100 \delta_{v, k} v_{k},
\end{aligned}
$$

where $\delta_{n, k}$ varies uniformly at each step on the unit interval for $n=x, w, v$. We also use $\bar{w}_{k}=0.1, \bar{v}_{k}=0.9, W_{k}=0.1$ and $V_{k}=2$ with initial conditions $\bar{x}_{0}=[21]^{T}$ and $X_{0}=0.1 I$.

The matrices associated to the uncertainties are given by

$$
\begin{aligned}
& H_{A, k}=\left[\begin{array}{c}
0 \\
10
\end{array}\right], H_{B w, k}=\left[\begin{array}{c}
0 \\
10
\end{array}\right], H_{B v, k}=\left[\begin{array}{l}
0 \\
0
\end{array}\right], \\
& H_{C, k}=50, H_{D w, k}=0, H_{D v, k}=100, \\
& G_{x, k}=\left[\begin{array}{ll}
0.1 & 0.03
\end{array}\right], G_{w, k}=0.01, G_{v, k}=1 .
\end{aligned}
$$

The scalar parameters are calculated at each step as

$$
\begin{aligned}
& \alpha_{x, k}^{-1}=\sigma_{\max }\left\{G_{x, k} \bar{P}_{k \mid k-1} G_{x, k}^{T}\right\}+\epsilon_{x}, \\
& \alpha_{w, k}^{-1}=\sigma_{\max }\left\{G_{w, k} W_{k} G_{w, k}^{T}\right\}+\epsilon_{w}, \\
& \alpha_{v, k}^{-1}=\sigma_{\max }\left\{G_{v, k} V_{k} G_{v, k}^{T}\right\}+\epsilon_{v},
\end{aligned}
$$

where $\sigma_{\max }\{\bullet\}$ indicates the maximum singular value of a matrix. Numerical simulations show that, in general, smaller values of $\epsilon_{x}, \epsilon_{w}$ and $\epsilon_{v}$ result in better bounds. However, this can lead to bad inverses calculation. In this example, we have chosen $\epsilon_{x}=\epsilon_{w}=\epsilon_{v}=0.1$. The mean value of the covariance matrices obtained over 500 experiments at $k=1500$ for the robust predictor and the classic predictor are

$$
\bar{P}_{\text {robust }}=\left[\begin{array}{cc}
14.4 & -22.7 \\
-22.7 & 76.4
\end{array}\right], \bar{P}_{\text {classic }}=\left[\begin{array}{cc}
3.6 & -0.6 \\
-0.6 & 0.1
\end{array}\right] \text {. }
$$

Fig. 2 shows the time evolution of the mean value (over 500 experiments) of both states and of their estimated values using the classic and the robust predictors.. It can be verified that the estimates of the classic predictor keep oscillating while the robust predictor reaches an approximate stationary value. The dynamics of the actual model also presents approximate stationary values for both state. It means that the robust predictor were able to better estimate the dynamics of the model.

The covariance ellipses obtained from both predictors and the actually obtained states at $k=1500$ are shown at Fig. 3. Although the size of the ellipse is smaller for the classic Kalman predictor, some states of the actual model are outside this bound. Fig. 4 presents the time evolution of the error variances for both states of the system. The error variances were approximated using the ensemble-average, defined in Sayed (2001).

The proposed filter reaches their approximate stationary states after a few steps while the Kalman filter did not. Fig. 4 also shows that the actual error variance of the proposed filter is always below its upper bound. Although the error variance of the Kalman filter is lower than the upper bound of the robust estimator, the actual error variance of the Kalman filter is 
above its error variance prediction, i.e., the Kalman filter does not guarantee the containment of the true signal $y_{k}$. This is a known result and it is presented in Ghaoui \& Clafiore (2001).
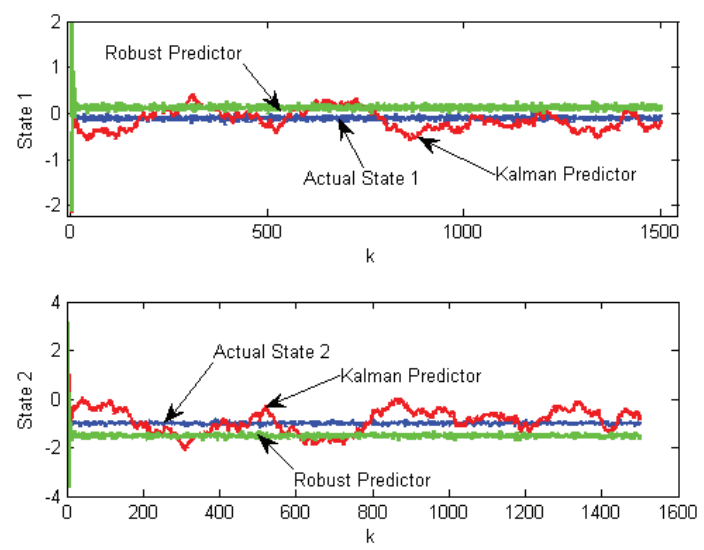

Fig. 2. Evolution of state 2 and its robust estimates.

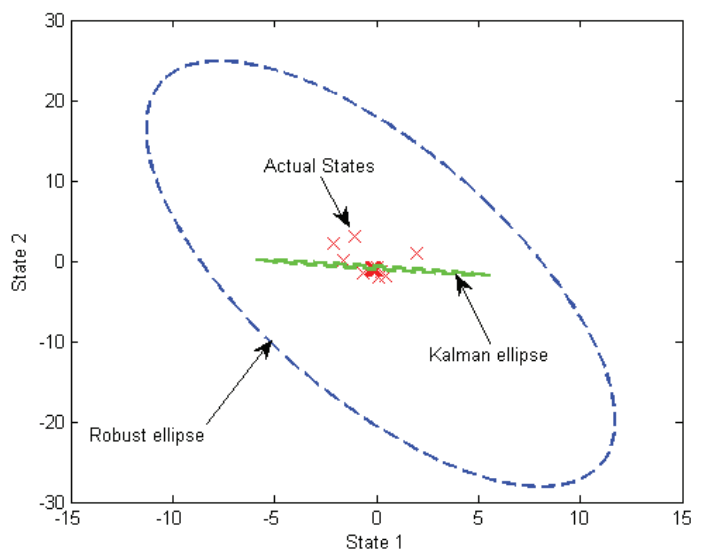

Fig. 3. Mean covariance ellipses after 1500 experiments.

A comparison with using the robust predictor presented here and another predictor found in the literature is shown at ????? The results presented therein show that the enhanced predictor presented here provides a less conservative design, with lower upper bound and lower experimental value of the error variance.

\section{Conclusions}

This chapter presented how to design robust predictor for linear systems with norm-bounded and time-varying uncertainties in their matrices. The design is based on a guaranteed cost 

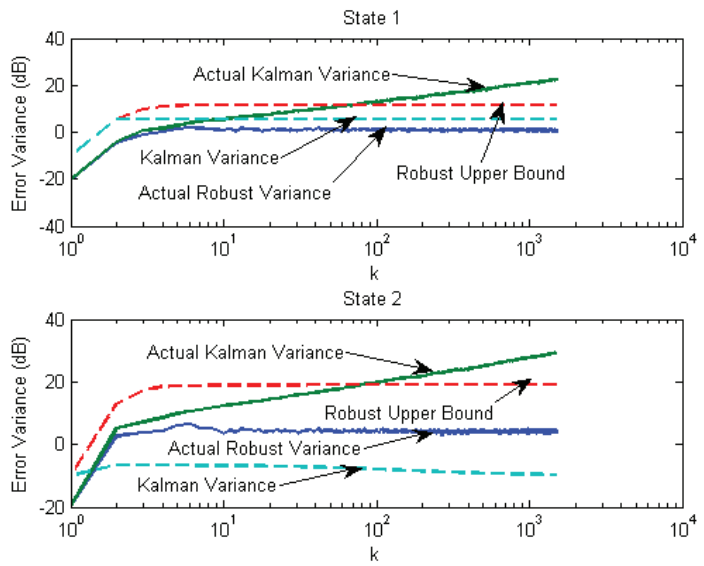

Fig. 4. Error variances for uncorrelated noise simulation.

approach using the Riccati equation. The obtained estimator is is capable of dealing with systems that present correlated dynamical and measurement noises with unknown mean and variance. In most of real life applications this is a common situation. It is also remarkable that the separated structure for the noises allows the estimator to have a less conservative upper bound for the covariance of the estimation error.

Further studies may include the use of approach of this chapter to design estimators for infinite time horizon discrete systems. Future studies may also investigate the feasibility to design a estimator for a more general description of systems: the descriptor systems.

\section{References}

Anderson, B. D. O. \& Moore, J. B. (1979). Optimal Filtering, Prentice-Hall.

Fu, M., de Souza, C. E. \& Luo, Z.-Q. (2001). Finite-horizon robust Kalman filter design, IEEE Transactions on Signal Processing 49(9): 2103-2112.

Ghaoui, L. E. \& Clafiore, G. (2001). Robust filtering for discrete-time systems with bounded noise and parametric uncertainty, IEEE Transactions on Automatic Control 46(7): 1084-1089.

Ishihara, J. Y., Terra, M. H. \& Campos, J. C. T. (2006). Robust Kalman filter for descriptor systems, IEEE Transactions on Automatic Control 51(8): 1354-1358.

Kalman, R. E. (1960). A New Approach to Linear Filtering and Prediction Problems, Transactions of the ASME 82 (1): 35-45.

Sayed, A. H. (2001). A framework for state space estimation with uncertain models, IEEE Transactions on Automatic Control 46(7): 998-1013.

Simon, D. (2006). Optimal State Estimation (2006). John Wiley and Sons.

Theodor, Y. \& Shaked, U. (1996). Robust discrete-time minimum variance filtering, IEEE Transactions on Signal Processing 44(2): 181-189.

Wang, Z., Zhu, J. \& Unbehauen, H. (1999). Robust filter design with time-varying parameter uncertainty and error variance constraints, International Journal of Control 72(1): 30-38. 


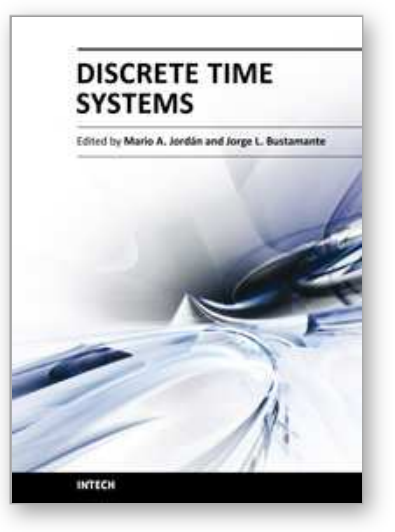

\author{
Discrete Time Systems \\ Edited by Dr. Mario Alberto Jord $\tilde{A}_{i n}$
}

ISBN 978-953-307-200-5

Hard cover, 526 pages

Publisher InTech

Published online 26, April, 2011

Published in print edition April, 2011

Discrete-Time Systems comprehend an important and broad research field. The consolidation of digital-based computational means in the present, pushes a technological tool into the field with a tremendous impact in areas like Control, Signal Processing, Communications, System Modelling and related Applications. This book attempts to give a scope in the wide area of Discrete-Time Systems. Their contents are grouped conveniently in sections according to significant areas, namely Filtering, Fixed and Adaptive Control Systems, Stability Problems and Miscellaneous Applications. We think that the contribution of the book enlarges the field of the Discrete-Time Systems with signification in the present state-of-the-art. Despite the vertiginous advance in the field, we also believe that the topics described here allow us also to look through some main tendencies in the next years in the research area.

\title{
How to reference
}

In order to correctly reference this scholarly work, feel free to copy and paste the following:

Rodrigo Souto, João Ishihara and Geovany Borges (2011). Kalman Filtering for Discrete Time Uncertain Systems, Discrete Time Systems, Dr. Mario Alberto JordÃ in (Ed.), ISBN: 978-953-307-200-5, InTech, Available from: http://www.intechopen.com/books/discrete-time-systems/kalman-filtering-for-discrete-timeuncertain-systems

\section{INTECH}

open science | open minds

\author{
InTech Europe \\ University Campus STeP Ri \\ Slavka Krautzeka 83/A \\ 51000 Rijeka, Croatia \\ Phone: +385 (51) 770447 \\ Fax: +385 (51) 686166 \\ www.intechopen.com
}

\author{
InTech China \\ Unit 405, Office Block, Hotel Equatorial Shanghai \\ No.65, Yan An Road (West), Shanghai, 200040, China \\ 中国上海市延安西路65号上海国际贵都大饭店办公楼 405 单元 \\ Phone: +86-21-62489820 \\ Fax: $+86-21-62489821$
}


(C) 2011 The Author(s). Licensee IntechOpen. This chapter is distributed under the terms of the Creative Commons Attribution-NonCommercialShareAlike-3.0 License, which permits use, distribution and reproduction for non-commercial purposes, provided the original is properly cited and derivative works building on this content are distributed under the same license. 